\title{
Conservation laws for even order systems of polyharmonic map type
}

\author{
Frédéric Louis de Longueville ${ }^{1} \cdot$ Andreas Gastel $^{2}$
}

Received: 12 September 2019 / Accepted: 31 March 2021 / Published online: 29 June 2021

(c) The Author(s) 2021

\begin{abstract}
Following Rivière's study of conservation laws for second order quasilinear systems with critical nonlinearity and Lamm/Rivière's generalization to fourth order, we consider similar systems of order $2 m$. Typical examples are $m$-polyharmonic maps. Under natural conditions, we find a conservation law for weak solutions on $2 m$-dimensional domains. This implies continuity of weak solutions.
\end{abstract}

Mathematics Subject Classification $58 \mathrm{E} 20 \cdot 35 \mathrm{~J} 35$

\section{Introduction}

The regularity of harmonic maps and related systems has been an active topic of research for decades. The harmonic map system in two dimensions has a critical nonlinearity, and in general such systems can have solutions that fail to be continuous. But not so for harmonic maps of surfaces. For a long time, it was not clear which are the general structural assumptions for critical nonlinear systems in two dimensions that allow proving full regularity of solutions.

The question was finally solved by Rivière [10] in 2007. The harmonic map system for harmonic maps $u: \mathbb{R}^{2} \supset U \rightarrow N$ is usually written as $\Delta u-\operatorname{tr} A(u)(d u, d u)=0$, where $A$ is the second fundamental form of the target manifold $N \subset \mathbb{R}^{n}$. Rivière re-interpreted the system as a linear system

$$
-\Delta u=\langle\Omega, d u\rangle,
$$

where $\Omega$ is a matrix-valued 1 -form depending on $x, u$, and $d u$. The point is that we can "forget" the dependence of $\Omega$ on $u$ and $d u$ once we have identified the important structural

Communicated by T. Rivière.

Andreas Gastel

andreas.gastel@uni-due.de

Frédéric Louis de Longueville

frederic.de-longueville@uni-due.de

1 Lehrstuhl Theoretische Informatik, Fakultät für Ingenieurwissenschaften, Universität Duisburg-Essen, 47048 Duisburg, Germany

2 Fakultät für Mathematik, Universität Duisburg-Essen, 45117 Essen, Germany 
assumptions we may impose on $\Omega$. The price one has to pay is that it has relatively little regularity. Since it depends linearly on $D u$, it will be a priori only in $L^{2}$. The bit of extra structure for $\Omega$ from the harmonic map system is that it can be rewritten in such a way that $\Omega$ takes its values in the skew-symmetric matrices. This is what is needed to prove continuity of weak solutions. We do not need to know the system is about harmonic maps-it is just the skew symmetry of $\Omega$ that enters the proof, which is much less of an assumption than what might have been expected before Rivière's discovery.

The continuity then follows from the fact that the system can be rewritten as a conservation law

$$
d(* A d u-(* B) \wedge d u)=0,
$$

provided that one can find functions $A \in W^{1,2} \cap L^{\infty}(U, G L(n))$ and $B \in W^{1,2}(U, \operatorname{so}(n) \otimes$ $\wedge^{2} \mathbb{R}^{2}$ ) satisfying

$$
d A-A \Omega=-\delta B
$$

For fourth order systems, the situation is quite similar. Regularity for weak solutions of critically nonlinear systems in four dimensions cannot be expected to hold in general. There are several variants of biharmonic map equations, for which regularity is known to hold. And in 2008, Lamm and Rivière [8] identified structural aspects of the different biharmonic systems that are responsible for continuity of weak solutions. Again, they rewrite the systems in the form of a linear equation

$$
\Delta^{2} u=\Delta\langle V, d u\rangle+\delta(w d u)+\langle W, d u\rangle
$$

where the coefficient functions $V \in W^{1,2}, w \in L^{2}$ and $W \in W^{-1,2}$ a priori have little regularity. But again, the least regular coefficient $W$ is actually slightly better, namely $W=$ $d \eta+F$ with $F \in L^{4 / 3,1}$ and $\eta \in L^{2}$ and $\eta$ skew-symmetric. Note that we have $L^{4 / 3} \hookrightarrow W^{-1,2}$ and that $F$ is slightly better.

Again, Lamm and Rivière find a conservation law

$$
\delta[d(A \Delta u)-2 d A \Delta u+\Delta A d u-A w d u+d A\langle V, d u\rangle-A d\langle V, d u\rangle-\langle B, d u\rangle]=0,
$$

provided that there are $A \in W^{2,2} \cap L^{\infty}(U, G L(n))$ and $B \in W^{1, \frac{4}{3}}\left(U, \mathbb{R}^{n \times n} \otimes \wedge^{2} \mathbb{R}^{4}\right)$ for which

$$
\Delta d A+(\Delta A) V-(d A) w+A W=\delta B .
$$

In both cases, the hard part of the regularity proof is to show that the auxiliary functions $A$ and $B$ exist under a smallness condition that can be satisfied by working on sufficiently small balls.

In this paper, we generalize the results from [10] $(m=1)$ and [8] $(m=2)$ to systems of order $2 m$ on a $2 m$-dimensional domain. The role that harmonic maps and biharmonic maps played so far, will now be taken by (extrinsically or intrinsically) polyharmonic maps. They are critical points $u: \mathbb{R}^{2 m} \supset U \rightarrow N \subset \mathbb{R}^{n}$ of the extrinsic polyharmonic energy $E_{m}(u):=\int_{U}\left|D^{m} u\right|^{2} d x$ or the intrinsic polyharmonic energy $E_{m}^{i}(u):=\int_{U}\left|\nabla^{m-1} d u\right|^{2} d x$. Again, weak solutions of the corresponding Euler-Lagrange equations are smooth, while the same cannot be expected for solutions of a general semilinear equation of order $2 m$ with critical nonlinearity. The structure needed to prove continuity of weak solutions along the lines of [10] and [8] turns out to be quite similar to what we learned above. This time, the 
formally linear equation reads

$$
\Delta^{m} u=\sum_{k=0}^{m-1} \Delta^{k}\left\langle V_{k}, d u\right\rangle+\sum_{k=0}^{m-2} \Delta^{k} \delta\left(w_{k} d u\right),
$$

where again we have coefficient functions that in the model case depend on $u$ and its derivatives, they are

$$
\begin{aligned}
& w_{k} \in W^{2 k+2-m, 2}\left(B^{2 m}, \mathbb{R}^{n \times n}\right) \quad \text { for } k \in\{0, \ldots, m-2\}, \\
& V_{k} \in W^{2 k+1-m, 2}\left(B^{2 m}, \mathbb{R}^{n \times n} \otimes \wedge^{1} \mathbb{R}^{2 m}\right) \quad \text { for } k \in\{0, \ldots, m-1\}, \text { where } \\
& V_{0}=d \eta+F, \\
& \eta \in W^{2-m, 2}\left(B^{2 m}, \operatorname{so}(n)\right), \quad F \in W^{2-m, \frac{2 m}{m+1}, 1}\left(B^{2 m}, \mathbb{R}^{n \times n} \otimes \wedge^{1} \mathbb{R}^{2 m}\right) .
\end{aligned}
$$

Note that the regularity assumption on the least regular coefficient $V_{0}$ is quite analogous to those made above. It is slightly better than the $V_{0} \in W^{2-m, 2}$ one would have in the general critical nonlinearity, in the sense that it decomposes in a skew-symmetric term and a term enjoying a tiny bit of extra (Lorentz) regularity. What we can gain from this are again a conservation law (see Theorem 4.1 below for the precise statement) and continuity of weak solutions (see Theorem 5.1).

However, the setting is slightly less pleasant here than in the previous cases, because we no longer have reduced everything to coefficients that are functions. Now $\eta$ and several other coefficients are in Sobolev spaces of negative order, which means they only make sense as distributions. This results in a conservation law that depends on a function $A \in W^{m, 2} \cap L^{\infty}$ and a distribution $B \in W^{2-m, 2}$ that satisfy some auxiliary equation. It is this difference between functions and distributions that makes our considerations more technical than those of [8]. Rather than splitting the auxiliary equation for $A$ and $B$ into a system of two equations, we have to solve a much larger system, roughly to determine all $B_{\alpha}$ in a decomposition $B=\sum_{|\alpha| \leq m-2} D^{\alpha} B_{\alpha}$.

Apart from that extra complication from dealing with negative differentiability, our methods are reasonably close to Rivière's and Lamm/Rivière's. In particular, we use Uhlenbeck's gauge theorem, Lorentz spaces and Hodge decomposition.

Regularity of weakly polyharmonic maps has already been proven by Scheven and the second author in [3]. It is not our primary focus here to re-prove that theorem, but to study the problem under minimal structural conditions on the equations as in the works by Rivière and Lamm/Rivière.

This paper is based on the first author's PhD thesis. Funded by the Deutsche Forschungsgemeinschaft (DFG, German Research Foundation) - 262992434.

\section{Preliminaries}

\subsection{Facts about Lorentz spaces}

Let $U \subset \mathbb{R}^{d}$ be a bounded domain with smooth boundary. Lorentz spaces $L^{p, q}(U)$ with $p, q \in[1, \infty]$ interpolate between the Lebesgue spaces $L^{p}(U)$ in such a way that $L^{p, p}(U)=L^{p}(U)$ and $L^{p, 1}(U) \subset \cdots \subset L^{p, p}(U) \subset \cdots \subset L^{p, \infty}(U)$ for all $p$. We refer to Ziemer's book [13, $\S 1.8$ and $\S 2.10]$ for definitions and basic properties. We are going to use embedding properties and Calderon-Zygmund estimates for Lorentz spaces rather than the actual definition. 
For $k \in \mathbb{N}$, we denote by $W^{k, p, q}(U)$ the Lorentz-Sobolev space of all functions $f$ on $U$ which are weakly differentiable $k$ times with $D^{\alpha} f \in L^{p, q}(U)$ for all $|\alpha| \leq k$. The $W^{k, p, q}$-norm is the sum of the $L^{p, q}$-norms of the $D^{\alpha} f$.

For $k \in \mathbb{Z}_{<0}, W^{k, p, q}$-spaces are often defined as duals of Sobolev-Lorentz spaces. But doing so, we would miss the spaces for $q=1$, which we will use throughout the paper. We therefore use the alternative definition, which is equivalent to the dual one in the $q>1$ and $p>1$ cases. For $k \in \mathbb{Z}_{<0}, p, q \in[1, \infty]$, we define $W^{k, p, q}(U)$ to be the space of all distributions on $U$ of the form $f=\sum_{|\alpha| \leq-k} D^{\alpha} f_{\alpha}$ with $f_{\alpha} \in L^{p, q}(U)$. The corresponding norm is defined as $\|f\|_{W^{k, p, q}(U)}:=\inf \sum_{|\alpha| \leq-k}\left\|f_{\alpha}\right\|_{L^{p, q}(U)}$, where the infimum is taken over all decompositions of $f$ as given in the definition.

Here are some facts about (Sobolev-)Lorentz spaces we are going to use.

(1) Sobolev-Lorentz embeddings They have been proven by Tartar [11] for Sobolev-Lorentz spaces on $\mathbb{R}^{d}$. Using extension theorems which hold analogous to the ones for usual Sobolev spaces, we easily carry them over to smooth bounded domains. If $k, \ell \in \mathbb{Z}, 1<p<\frac{d}{\ell}$ and $1 \leq q \leq \infty$, then every $f \in W^{k, p, q}(U)$ is also in $W^{k-\ell, \frac{d p}{d-\ell p}, q}(U)$ with

$$
\|f\|_{W^{k-\ell, \frac{d p}{d-\ell p}, q}(U)} \leq C\|f\|_{W^{k, p, q}(U)} .
$$

(2) Multiplication theorems Assume $f \in W^{p, q}(U)$ and $g \in W^{p^{\prime}, q^{\prime}}(U)$, where $1<p, p^{\prime}<$ $\infty$ with $\frac{1}{p}+\frac{1}{p^{\prime}}<1$ and $1 \leq q, q^{\prime} \leq \infty$. Then

$$
\|f g\|_{L^{r, s}} \leq C\|f\|_{L^{p, q}}\|g\|_{L^{p^{\prime}, q^{\prime}}}
$$

if $\frac{1}{r}=\frac{1}{p}+\frac{1}{p^{\prime}}$ and $\frac{1}{s} \leq \frac{1}{q}+\frac{1}{q^{\prime}}, s \geq 1$. In the boundary case $\frac{1}{p}+\frac{1}{p^{\prime}}=1$, we have

$$
\|f g\|_{L^{1}} \leq\|f\|_{L^{p, q}}\|g\|_{L^{p^{\prime}, q^{\prime}}}
$$

whenever $\frac{1}{q}+\frac{1}{q^{\prime}} \leq 1$. See [9, Thm. 3.4, 3.5] for proofs. From these theorems together with the Sobolev-Lorentz embeddings above, we also have Sobolev-Lorentz multiplication theorems. Suppose $f \in W^{k, p, q}(U)$ and $g \in W^{z, p^{\prime}, q^{\prime}}$ with $k \in \mathbb{N} \cup\{0\}, z \in \mathbb{Z}, 1<p, p^{\prime}<\infty, 1 \leq$ $q, q^{\prime}<\infty$. If $\frac{1}{p}+\frac{1}{p^{\prime}} \leq 1, k \geq|z|$, and $k p<d$, then $f g \in W^{z, x, y}(U)$ for $x:=\frac{d p p^{\prime}}{d\left(p+p^{\prime}\right)-k p p^{\prime}}$ and $\frac{1}{y}:=\min \left\{1, \frac{1}{q}+\frac{1}{q^{\prime}}\right\}$, and

$$
\|f g\|_{W^{z, y, x}} \leq C\|f\|_{W^{k, p, q}}\|g\|_{W^{z, p^{\prime}, q^{\prime}}} .
$$

The assertion continues to hold in the case $k p=d$ (and then with $x=p^{\prime}$ and $y=q^{\prime}$ ) if we additionally assume $f \in L^{\infty}$.

(3) $L^{p}$ theory The same way we have $W^{k+2, p}$ estimates for solutions of $\Delta u=f \in W^{k, p}$ with suitable boundary data, we also have $W^{k+2, p, q}$-estimates if $\Delta u=f \in W^{k, p, q}$. This is proven by standard interpolation arguments and was used extensively in Rivière's and Lamm's paper. Similarly, Hodge decomposition theorems in $W^{k, p}$ extend to $W^{k, p, q}$. Using our definition of $W^{k, p, q}$ for $k<0$, we can even extend most of the results to those spaces by applying them to a decomposition $f=\sum_{|\alpha| \leq-k} D^{\alpha} f_{\alpha}$. We have to be careful with boundary values, though, imposing them on the "partial solutions" $u_{\alpha}$ of $\Delta u_{\alpha}=f_{\alpha}$ and finally defining $u:=\sum_{|\alpha| \leq-k} D^{\alpha} u_{\alpha}$. Reducing any solution of $\Delta f=g$ to a solution of such boundary value problems using cutoff functions, it is standard to prove the following.

Lemma 2.1 (Some basic $L^{p, q}$-theory) Assume $k \in \mathbb{Z}, p \in(1, \infty), q \in[1, \infty)$. Given $g \in W^{k, p, q}\left(B_{1}\right)$, any weak solution $f$ of

$$
\Delta f=g
$$


is in $W^{k+2, p, q}\left(B_{1 / 2}\right)$, and we have

$$
\|f\|_{W^{k+2, p, q}\left(B_{1 / 2}\right)} \leq c\|g\|_{W^{k, p, q}\left(B_{1}\right)}
$$

with a constant $c$ depending only on $k, p, q$ and the domain dimension.

(4) A nontrivial Hodge-style Lemma The Lemma that follows is taken from [10, Lemma A.2] for $m=1$ and [8, Lemma A.2] for $m=2$. Their proof carries over immediately. Even though the assertion looks little surprising in view of Hodge decompositions, it must be emphasized that this is one of the least trivial ingredients in this paper. The proof does involve the Coifman-Lions-Meyer-Semmes-Lemma [1], Hardy-BMO-duality, and the fact that the Neumann problem for the Poisson equation with right-hand side in $\mathcal{H}^{1}\left(\mathbb{R}^{d}\right)$ can be solved in $W^{1, \frac{d}{d-1}, 1}\left(\mathbb{R}^{d}\right)$. That is, the Lemma uses all the facts about "exotic" function spaces that have been found to be important for harmonic map theory by Hélein [7] and many others.

Lemma 2.2 Assume $m \in \mathbb{N}$. There is $\varepsilon>0$ such that for all $Q \in W^{m, 2} \cap L^{\infty}\left(B^{2 m}, S O(n)\right)$ satisfying $Q-I \in W_{0}^{m, 2}$ and $\|d Q\|_{L^{2 m}\left(B^{2 m}\right)}<\varepsilon$, the boundary value problem

$$
\begin{cases}d[(\delta C) Q]=0 & \text { on } B^{2 m}, \\ d C=0 & \text { on } B^{2 m}, \\ C_{N}=0 & \text { on } \partial B^{2 m}\end{cases}
$$

in $W^{1, \frac{2 m}{2 m-1}}$ has the trivial solution $C \equiv 0$ as the only solution.

(5) Continuity of $W^{k, d / k, 1}$-functions An important point in proving continuity of our weak solutions will be to prove that they are in $W^{m+1, \frac{2 m}{m+1}, 1}$ and then use that this space embeds into $C^{0}$. This comes from an embedding which generalizes the well-known embedding $W^{d, 1} \rightarrow$ $C^{0}$. It was used, but not explicitly stated, in [8, Section 2.3] for $d=4, k=2$. Our proof uses the arguments presented there.

Theorem 2.3 Let $\Omega \subset \mathbb{R}^{d}$ be any bounded domain of class $C^{k+1}$, and assume $k \in\{1, \ldots, d\}$. Then any map $u \in W^{k, d / k, 1}(\Omega)$ is also in $C^{0}(\Omega)$ and obeys the estimate

$$
\|u\|_{L^{\infty}(\Omega)} \leq c\|u\|_{W^{k, d / k, 1}(\Omega)} .
$$

Proof Since the embedding $W^{d, 1,1}=W^{d, 1} \hookrightarrow C^{0}$ is well-known, we may assume $k<d$. Assume that $k$ is odd. Let $G$ be the fundamental solution of $\Delta^{(k+1) / 2}$ on $\mathbb{R}^{d}, f:=d G$, which means $f(x)=c|x|^{k-d-1} x$ and hence $f \in L^{d /(d-k), \infty}(\Omega)=L^{d / k, 1}(\Omega)^{*}$. Extend $u$ to a function $u \in W^{k, d / k, 1}\left(\mathbb{R}^{d}\right)$ with norm controlled by the one on the original $u$. For any $x \in \mathbb{R}^{d}$, we have

$$
\begin{aligned}
|u(x)| & =\left|\int_{\mathbb{R}^{d}} \Delta^{(k+1) / 2} G(x-y) u(y) d x\right|=\left|\int_{\mathbb{R}^{d}}\left\langle f(x-y), d \Delta^{(k-1) / 2} u(y)\right\rangle d y\right| \\
& \leq\|f\|_{L^{d /(d-k), \infty}\left(\mathbb{R}^{d}\right)}\left\|d \Delta^{(k-1) / 2} u\right\|_{L^{d / k, 1}\left(\mathbb{R}^{d}\right)} \leq c\|u\|_{W^{k, d / k, 1}(\Omega)},
\end{aligned}
$$

which proves the $L^{\infty}$-estimate. Now approximate $u$ in $W^{k, d / k, 1}\left(\mathbb{R}^{d}\right)$ by functions $u_{j} \in$ $C_{c}^{\infty}\left(\mathbb{R}^{d}\right)$. By the estimate just proven, $\left\|u_{j}-u_{i}\right\|_{L^{\infty}\left(\mathbb{R}^{d}\right)} \leq c\left\|u_{j}-u_{i}\right\|_{W^{k, d / k, 1}\left(\mathbb{R}^{n}\right)}$, which shows that $\left(u_{j}\right)$ is a uniform Cauchy sequence, implying continuity of $u$.

If $k$ is even, a similar reasoning uses the fundamental solution $G$ for $\Delta^{k / 2} \delta$ and $f:=\delta G$. 


\subsection{Uhlenbeck decomposition}

We will need a suitable adaptation of Uhlenbeck's gauge theorem [12, Theorem 1.3] in the spirit of Rivière's reinterpretation [10, Lemma A.4], cf. [8, Theorem A.5] for the $m=2$ case. See also [4] for a discussion of its analytic aspects.

Theorem 2.4 Assume that $m, n \in \mathbb{N}$ and $B_{r} \subset \mathbb{R}^{2 m}$ is a ball of radius $r$. Then there is $\varepsilon>0$ such that for all $\Omega \in W^{m-1,2}\left(B_{r}, \operatorname{so}(n) \otimes \wedge^{1} \mathbb{R}^{2 m}\right)$ satisfying $\|\Omega\|_{W^{m-1,2}\left(B_{r}\right)}<\varepsilon$, there are functions $P \in W^{m, 2}\left(B_{r / 2}, S O(n)\right)$ and $\Phi \in W^{m, 2}\left(B_{r / 2}\right.$, so $\left.(n) \otimes \wedge^{2} \mathbb{R}^{2 m}\right)$ such that

$$
\Omega=P d P^{-1}+P \delta \Phi P^{-1}
$$

holds on $B_{r / 2}$. Moreover, we have the estimate

$$
\|d P\|_{W^{m-1,2}\left(B_{r / 2}\right)}+\|\delta \Phi\|_{W^{m-1,2}\left(B_{r / 2}\right)} \leq c\|\Omega\|_{W^{m-1,2}\left(B_{r}\right)} .
$$

Proof The proof is given in the references above, except for the estimates which we find there only for $\|d P\|_{W^{1,2}}$ and $\|\delta \Phi\|_{W^{1,2}}$. We can have $d \Phi=0$ there, and bootstrapping the estimates through the equations $d \Phi=0$ and $P^{-1} d P^{-1}+P^{-1} \Omega P=\delta \Phi$ easily gives the higher order estimates.

\section{Polyharmonic maps and their equations}

\subsection{Extrinsically polyharmonic maps}

Extrinsically polyharmonic maps $u: \mathbb{R}^{n} \supset U \rightarrow N$ are critical points of $\int\left|D^{m} u\right|^{2} d x$. The most natural way to write down the Euler-Lagrange equation for that functional is

$$
\Delta^{m} u \perp N .
$$

To make this an explicit system of differential equations, we use local smooth orthonormal bases $\left\{v_{i}(u(x))\right\}$ of $T_{u(x)}^{\perp} N$ and can therefore write

$$
\Delta^{m} u=\sum_{i} \lambda_{i}\left(v_{i} \circ u\right)
$$

with Lagrange multipliers $\lambda_{i}$ that we are going to determine. Multiplying with $(v \circ u)$ and using Leibniz' rule iteratively, we find

$$
\begin{aligned}
\lambda_{i} & =\left\langle\Delta^{m} u, v_{i} \circ u\right\rangle \\
& =\Delta^{m}\left\langle u, v_{i} \circ u\right\rangle-\sum_{t=0}^{m-1} \Delta^{t}\left\langle d \Delta^{m-t-1} u, J_{i} d u\right\rangle-\sum_{t=0}^{m-1} \Delta^{t} \delta\left\langle\Delta^{m-t-1} u, J_{i} d u\right\rangle,
\end{aligned}
$$

where here $J_{i}:=d v_{i} \circ u$. Expanding the innermost $\Delta=\delta d$, the first term on the right-hand side becomes

$$
\Delta^{m}\left\langle u, v_{i} \circ u\right\rangle=\Delta^{m-1} \delta\left[\left\langle d u, v_{i} \circ u\right\rangle+\left\langle u, J_{i} d u\right\rangle\right]=\Delta^{m-1} \delta\left\langle u, J_{i} d u\right\rangle,
$$

since $d u$ is tangential to $N$ and $v_{i} \circ u$ is normal. Hence

$$
\lambda_{i} v_{i}=-\sum_{t=0}^{m-1} \Delta^{t}\left\langle d \Delta^{m-t-1} u, J_{i} d u\right\rangle \nu_{i}-\sum_{t=0}^{m-2} \Delta^{t} \delta\left\langle\Delta^{m-t-1} u, J_{i} d u\right\rangle \nu_{i},
$$


where we have abbreviated $v_{i} \circ u$ by $v_{i}$ and will keep doing so. The $t=0$ term in the first sum can be rewritten as

$$
-\left\langle\Delta^{m-1} d u, J_{i} d u\right\rangle v_{i}=-\left\langle v_{i} \otimes J_{i}^{T} \Delta^{m-1} d u, d u\right\rangle
$$

while for $t>0$ a long but straightforward calculation shows

$$
\begin{aligned}
-\Delta^{t}\left\langle\Delta^{m-t-1} d u, J_{i} d u\right\rangle v_{i}= & -\Delta^{t}\left\langle v_{i} \otimes J_{i}^{T} \Delta^{m-t-1} d u, d u\right\rangle \\
& +\sum_{j=1}^{t} \Delta^{t-j}\left\langle J_{i}^{T} \Delta^{j-1} d\left\langle\Delta^{m-t-1} d u, J_{i} d u\right\rangle, d u\right\rangle \\
& +\sum_{j=1}^{t} \Delta^{t-1} \delta\left[\Delta^{j-1}\left\langle\Delta^{m-t-1} d u, J_{i} d u\right\rangle J_{i} d u\right]
\end{aligned}
$$

The $t=0$ term in the second sum of (2) becomes

$$
-\delta\left\langle\Delta^{m-1} u, J_{i} d u\right\rangle v_{i}=-\delta\left\langle v_{i} \otimes J_{i}^{T} \Delta^{m-1} u, d u\right\rangle+\left\langle J_{i}^{T}\left\langle\Delta^{m-1} u, J_{i} d u\right\rangle, d u\right\rangle,
$$

while for $t>0$ we have

$$
\begin{aligned}
& -\Delta^{t} \delta\left\langle\Delta^{m-t-1} u, J_{i} d u\right\rangle v_{i}=-\Delta^{t} \delta\left\langle v_{i} \otimes J_{i}^{T} \Delta^{m-t-1} u, d u\right\rangle \\
& +\Delta^{t}\left\langle\left\langle J_{i} \Delta^{m-t-1} u, J_{i} d u\right\rangle, d u\right\rangle+\sum_{j=1}^{t} \Delta^{t-j}\left\langle J_{i}^{T} \Delta^{j-1} d \delta\left\langle\Delta^{m-t-1} u, J_{i} d u\right\rangle, d u\right\rangle \\
& +\sum_{j=1}^{t} \Delta^{t-j} \delta\left\langle\Delta^{j-1} \delta\left\langle\Delta^{m-t-1} u, J_{i} d u\right\rangle, J_{i} d u\right\rangle
\end{aligned}
$$

Inserting that into (2), we find

$$
\Delta^{m} u=\sum_{k=0}^{m-1} \Delta^{k}\left\langle V_{k}, d u\right\rangle+\sum_{k=0}^{m-2} \Delta^{k} \delta\left(w_{k} d u\right)
$$

with (abbreviating $d^{\ell}:=d \delta d \ldots$ and $\delta^{\ell}:=\delta d \delta \ldots$ with $\ell$ letters each)

$$
\begin{aligned}
V_{k}:= & \sum_{i}\left(-\left(v_{i} \circ u\right) \otimes J_{i}^{T} \Delta^{m-k-1} d u+\sum_{t=k+1}^{m-1} J_{i}^{T} \Delta^{t-k-1} d\left\langle\Delta^{m-t-1} d u, J_{i} d u\right\rangle\right. \\
& \left.+\sum_{t=k}^{m-2} J_{i}^{T} d^{2 t-2 k}\left\langle\Delta^{m-t-1} u, J_{i} d u\right\rangle\right) \\
w_{k}:= & \sum_{i}\left(-\left(v_{i} \circ u\right) \otimes J_{i}^{T} \Delta^{m-k-1} u+\sum_{t=k+1}^{m-1} J_{i}^{T} \Delta^{t-k-1}\left\langle\Delta^{m-t-1} d u, J_{i} d u\right\rangle\right. \\
& \left.+\sum_{t=k}^{m-2} J_{i}^{T} \delta^{2 t-2 k}\left\langle\Delta^{m-t-1} u, J_{i} d u\right\rangle\right)
\end{aligned}
$$

For weakly polyharmonic mappings, we must assume $u \in W^{m, 2}$, and then some of the terms in $V_{k}$ and $w_{k}$ exist only in the sense of distributions. But it is easily checked using 
Section 2.1(1)-(2) that the terms do not involve products of distributions (which would be undefined), and that

$$
\begin{gathered}
V_{k} \in W^{2 k+1-m, 2} \quad \text { for } k=0, \ldots, m-1, \\
w_{k} \in W^{2 k+1-m, 2} \text { for } k=0, \ldots, m-2 .
\end{gathered}
$$

But we will need that $V_{0}$ is better than that, and it actually can be improved. This is the important extra structure that Rivière discovered to be essential in [10] for second-order systems (and in [8] for fourth order). We can modify (3) by adding a term that is 0 because of $v_{i} \perp d u$,

$$
\begin{aligned}
& -\sum_{i}\left\langle\Delta^{m-1} d u, J_{i} d u\right\rangle v_{i} \\
& =\sum_{i}\left\langle J_{i}^{T} \Delta^{m-1} d u \otimes v_{i}-v_{i} \otimes J_{i}^{T} \Delta^{m-1} d u, d u\right\rangle \\
& =\sum_{i}\left\langle d\left[J_{i}^{T} \Delta^{m-1} u \otimes v_{i}-v_{i} \otimes J_{i}^{T} \Delta^{m-1} u\right], d u\right\rangle+\langle R, d u\rangle \\
& =:\langle d \eta, d u\rangle+\langle R, d u\rangle,
\end{aligned}
$$

where here $\eta$ takes its values in the set $s o(n)$ of skew-symmetric $n \times n$-matrices, and $\eta \in$ $W^{2-m, 2}$. The remaining terms in $V_{0}$, including new ones from $R$, are all products of at least two $W^{\cdots, 2}$-functions, hence they are in some Sobolev-Lorentz space $W^{\cdots, \cdots, \ldots, 1}$. A case-by-case inspection of all summands shows that our modified $V_{0}$ splits into

$$
V_{0}=d \eta+F, \quad \eta \in W^{2-m, 2}(U, s o(n)), \quad F \in W^{2-m, \frac{2 m}{m+1}, 1}\left(U, \mathbb{R}^{n \times n} \otimes \wedge^{1} \mathbb{R}^{2 m}\right) .
$$

This is as much structure as we will need for our regularity theory.

\subsection{Intrinsically polyharmonic maps}

Intrinsically polyharmonic maps are critical points of $\int\left|\nabla^{m-1} d u\right|^{2} d x$. Since

$$
\nabla^{m-1} d u-D^{m} u=\sum_{\mathbf{m} \in M_{1}}\left(X_{\mathbf{m}} \circ u\right)\left(D^{m_{1}} u, \ldots, D^{m_{\ell}} u\right)
$$

for suitable multilinear forms $X_{\mathbf{m}}$ and

$$
M_{1}:=\left\{\mathbf{m}=\left(m_{1}, \ldots, m_{\ell}\right) \in \bigcup_{\ell=2}^{m} \mathbb{N}^{\ell}: m_{1}+\cdots+m_{\ell}=m, m_{1} \geq \cdots \geq m_{\ell}\right\},
$$

the difference between the extrinsic and the intrinsic integrand can be written as

$$
\left|\nabla^{m-1} d u\right|^{2}-\left|D^{m} u\right|^{2}=\sum_{\mathbf{m} \in M_{2}}\left(Y_{\mathbf{m}} \circ u\right)\left(D^{m_{1}} u, \ldots, D^{m_{\ell}} u\right)
$$

with new multilinear forms $Y_{\mathbf{m}}$ and

$$
\begin{gathered}
M_{2}:=\left\{\mathbf{m}=\left(m_{1}, \ldots, m_{\ell}\right) \in \bigcup_{\ell=3}^{m}\{1, \ldots, m\}^{\ell}:\right. \\
\left.m_{1}+\cdots+m_{\ell}=2 m, m_{1} \geq \cdots \geq m_{\ell}\right\} .
\end{gathered}
$$


This means that the Euler-Lagrange equation for intrinsically polyharmonic maps reads (somewhat symbolically, since we do not indicate precisely which contractions are performed by the div-operators)

$$
\Delta^{m} u+\sum_{\mathbf{m} \in M_{3}} \operatorname{div}^{m_{1}}\left(\left(Z_{\mathbf{m}} \circ u\right)\left(D^{m_{2}} u, \ldots, D^{m_{\ell}} u\right) \perp N,\right.
$$

with yet more multilinear forms $Z_{\mathbf{m}}$ and

$$
\begin{gathered}
M_{3}:=\left\{\mathbf{m}=\left(m_{1}, \ldots, m_{\ell}\right) \in \bigcup_{\ell=3}^{m}\{1, \ldots, m\}^{\ell}:\right. \\
\left.m_{1}+\cdots+m_{\ell}=2 m, m_{2} \geq \cdots \geq m_{\ell}\right\} .
\end{gathered}
$$

It is now straightforward to check that the additional terms contribute to more summands for $V_{0}, \ldots, V_{m-1}, w_{0}, \ldots, w_{m-2}$ (in the same spaces) in the previous calculation, and that $\ell \geq 3$ in each summand implies that no derivatives of order $2 m-1$ will be involved in the terms contributing to $V_{0}$. Hence none of those will contribute to $d \eta$. This means that the Euler-Lagrange equation for intrinsically polyharmonic maps can be written in the form (4) with exactly the same regularity conditions for the coefficient functions as in the extrinsic case.

\subsection{Further equations}

The equations allowed here are by no means restricted to a geometric or variational context. Equations of the form (4) do not refer to a target manifold $N$ explicitly. For example, they include equations like the "fake polyharmonic equation"

$$
\Delta^{m} u+|D u|^{2 m} u=0,
$$

whose coefficient functions fulfill the required estimates if $u$ is bounded.

\section{A conservation law}

The following theorem reformulates a rather general system of order $2 m$ as a conservation law, in the spirit of Rivière's original idea [10, Theorems I.3 and I.4] $(m=1)$ and Lamm/Rivière's fourth order generalization [8, Theorems 1.3 and 1.5] $(m=2)$. We work on the unit ball $B^{2 m}$ of $\mathbb{R}^{2 m}$ and later use a scaling argument.

Theorem 4.1 Assume $m \geq 3, n \in \mathbb{N}$. Let coefficient functions be given as

$$
\begin{aligned}
w_{k} & \in W^{2 k+2-m, 2}\left(B^{2 m}, \mathbb{R}^{n \times n}\right) \quad \text { for } k \in\{0, \ldots, m-2\}, \\
V_{k} & \in W^{2 k+1-m, 2}\left(B^{2 m}, \mathbb{R}^{n \times n} \otimes \wedge^{1} \mathbb{R}^{2 m}\right) \quad \text { for } k \in\{0, \ldots, m-1\}, \text { where } \\
V_{0} & =d \eta+F, \\
\eta & \in W^{2-m, 2}\left(B^{2 m}, \operatorname{so}(n)\right), \quad F \in W^{2-m, \frac{2 m}{m+1}, 1}\left(B^{2 m}, \mathbb{R}^{n \times n} \otimes \wedge^{1} \mathbb{R}^{2 m}\right) .
\end{aligned}
$$

We consider the equation

$$
\Delta^{m} u=\sum_{k=0}^{m-1} \Delta^{k}\left\langle V_{k}, d u\right\rangle+\sum_{k=0}^{m-2} \Delta^{k} \delta\left(w_{k} d u\right) .
$$


For this equation, the following statements hold.

(i) Let

$$
\begin{gathered}
\theta:=\sum_{k=0}^{m-2}\left\|w_{k}\right\|_{W^{2 k+2-m, 2}\left(B^{2 m}\right)}+\sum_{k=1}^{m-1}\left\|V_{k}\right\|_{W^{2 k+1-m, 2}\left(B^{2 m}\right)} \\
\quad+\|\eta\|_{W^{2-m, 2}\left(B^{2 m}\right)}+\|F\|_{W^{2-m, \frac{2 m}{m+1}, 1}\left(B^{2 m}\right)} .
\end{gathered}
$$

There is $\theta_{0}>0$ such that whenever $\theta<\theta_{0}$, there are a function $A \in W^{m, 2} \cap$ $L^{\infty}\left(B_{1 / 4} ; G L(n)\right)$ and a distribution $B \in W^{2-m, 2}\left(B_{1 / 4}, \mathbb{R}^{n \times n} \otimes \wedge^{2} \mathbb{R}^{2 m}\right)$ that solve

$$
\Delta^{m-1} d A+\sum_{k=0}^{m-1}\left(\Delta^{k} A\right) V_{k}-\sum_{k=0}^{m-2}\left(\Delta^{k} d A\right) w_{k}=\delta B .
$$

(ii) A function $u \in W^{m, 2}\left(B_{1 / 2}, \mathbb{R}^{n}\right)$ solves (8) weakly on $B_{1 / 4}$ if and only if it is a distributional solution of the conservation law

$$
\begin{aligned}
& 0=\delta\left[\sum_{\ell=0}^{m-1}\left(\Delta^{\ell} A\right) \Delta^{m-\ell-1} d u-\sum_{\ell=0}^{m-2}\left(d \Delta^{\ell} A\right) \Delta^{m-\ell-1} u\right. \\
&-\sum_{k=0}^{m-1} \sum_{\ell=0}^{k-1}\left(\Delta^{\ell} A\right) \Delta^{k-\ell-1} d\left\langle V_{k}, d u\right\rangle+\sum_{k=0}^{m-1} \sum_{\ell=0}^{k-1}\left(d \Delta^{\ell} A\right) \Delta^{k-\ell-1}\left\langle V_{k}, d u\right\rangle \\
&-\sum_{k=0}^{m-2} \sum_{\ell=0}^{k-2}\left(\Delta^{\ell} A\right) d \Delta^{k-\ell-1} \delta\left(w_{k} d u\right)+\sum_{k=0}^{m-2} \sum_{\ell=0}^{k-2}\left(d \Delta^{\ell} A\right) \Delta^{k-\ell-1} \delta\left(w_{k} d u\right) \\
&-\langle B, d u\rangle] .
\end{aligned}
$$

(Here $d \Delta^{-1} \delta$ means the identity map.)

(iii) Every weak solution of (8) on $B^{2 m}$ is continuous on $B_{1 / 16}$ if the smallness condition $\theta<\theta_{0}$ holds.

Proof of Theorem 4.1 (ii) This can be done by direct calculation. A line-by-line calculation of the terms in (10) gives

$$
\begin{aligned}
\delta[\ldots]= & \left\langle d \Delta^{m-1} A, d u\right\rangle+A \Delta^{m} u \\
& -\sum_{k=0}^{m-1}\left\{A \Delta^{k}\left\langle V_{k}, d u\right\rangle-\left(\Delta^{k} A\right)\left\langle V_{k}, d u\right\rangle\right\} \\
& -\sum_{k=0}^{m-2}\left\{\left\langle d \Delta^{k} A, w_{k} d u\right\rangle+A \Delta^{k} \delta\left(w_{k} d u\right)\right\} \\
& -\langle\delta B, d u\rangle \\
= & A\left\{\Delta^{m} u-\sum_{k=0}^{m-1} \Delta^{k}\left\langle V_{k}, d u\right\rangle-\sum_{k=0}^{m-2} \Delta^{k} \delta\left(w_{k} d u\right)\right\} .
\end{aligned}
$$

Now (8) means that the last $\{\ldots\}$ vanishes, and $A$ is invertible by (i).

Proof of Theorem 4.1 (iii) On $B_{1 / 4}$, the conservation law (10) can be rewritten as

$$
0=\delta\left[A \Delta^{m-1} d u+(d A) \Delta^{m-1} u+R\right]=\Delta\left(A \Delta^{m-1} u\right)+\delta R,
$$


where here $R \in W^{2-m, \frac{2 m}{m+1}, 1}\left(B_{1 / 4}, \mathbb{R}^{n}\right)$. By $L^{p, q}$ theory in Lorentz-spaces as formulated in Lemma (2.1), we first have $A \Delta^{m-1} u \in W^{3-m, \frac{2 m}{m+1}, 1}$ on $B_{1 / 8}$. Invertibility of $A$ and $A \in W^{m, 2} \cap L^{\infty}$ give $\Delta^{m-1} u \in W^{3-m, \frac{2 m}{m+1}, 1}$ by the remark following (1), and therefore $u \in W^{m+1, \frac{2 m}{m+1}, 1}$ on $B_{1 / 16}$. But the latter space embeds into $C^{0}$ by Theorem 2.3 , which implies the asserted continuity of $u$.

Proof of Theorem 4.1 (i) This is the technical part of our paper. By iteratively solving Neumann problems on $B^{2 m}$ (in the case of negative Sobolev exponents to be understood in the sense described in Section 2.1(3)), we find $\Psi \in W^{m, 2}\left(B^{2 m}\right.$, so(n)) satisfying $\Delta^{m-1} \Psi=-\eta$. Note that we may assume $\int_{B^{2 m}} \eta d x=0$ since our $\eta$ enters into the theorem only in $d \eta$. Letting $\Omega:=d \Psi \in W^{m-1,2}\left(B^{2 m}, \operatorname{so}(n) \otimes \wedge^{1} \mathbb{R}^{2 m}\right)$, we have found $\Omega$ satisfying

$$
\Delta^{m-2} \delta \Omega=-\eta, \quad\|\Omega\|_{W^{m-1,2}\left(B^{2 m}\right)} \leq c\|\eta\|_{W^{2-m, 2}\left(B^{2 m}\right)} .
$$

If $\theta$ in our theorem has been chosen small enough, then $\Omega$ satisfies the smallness assumption for Theorem 2.4. Then we find corresponding functions $P$ and $\Phi$ such that

$$
V_{0}=d \eta+F=-d \Delta^{m-2} \delta \Omega+F=-d \Delta^{m-2} \delta\left(P d P^{-1}+P \delta \Phi P^{-1}\right)+F
$$

on $B_{1 / 2}$, where here $d P$ and $\delta \Phi$ are small on $B_{1 / 2}$ in their respective norms which are bounded by $c \theta$. We rewrite that further,

$$
V_{0}=-P d \Delta^{m-1} P^{-1}+K
$$

where here

$$
\begin{aligned}
K= & -d \Delta^{m-2}\left(\left\langle d P, d P^{-1}\right\rangle+\langle d P, \delta \Phi\rangle P^{-1}+P\left\langle\delta \Phi, d P^{-1}\right\rangle\right) \\
& +\sum_{j=1}^{2 m-3}\left\langle D^{j} P, D^{2 m-3-j} \Delta P^{-1}\right\rangle \\
\in & W^{2-m, \frac{2 m}{m+1}, 1}\left(B_{1 / 2}\right),
\end{aligned}
$$

and the corresponding norm is bounded by $c \theta$. Here and in the sequel, we use $\left\langle D^{a} U, D^{b} V\right\rangle$ as a symbol for any bilinear form involving $a$-th derivatives of $U$ and $b$-th derivatives of $V$, if it can be computed in principle and the explicit form does not matter.

We use the function $P$ constructed here to substitute $A$ by $(I+\widetilde{A}) P^{-1}$ and find that (9) transforms to an equation for $(\widetilde{A}, B)$ of the form

$$
d \Delta^{m-1} \widetilde{A}+\sum_{j=0}^{2 m-2}\left\langle D^{j} \widetilde{A}, K_{j}\right\rangle+K_{0}=\delta B P
$$

with coefficient functions $K_{0}, \ldots, K_{m-2}$ bounded by $c \theta$ in the norms of the spaces

$$
K_{0} \in W^{2-m, \frac{2 m}{m+1}, 1}\left(B_{1 / 2}\right), \quad K_{j} \in W^{j+1-m, 2}\left(B_{1 / 2}\right) \text { for } j \in\{1, \ldots, m-2\} .
$$

Now assume that instead of $w_{k}, V_{k}, \eta$, and $F$, we have started with $\psi w_{k}, \psi V_{k}, \psi \eta$, and $\psi F$ for some smooth $B_{1 / 4}-B_{1 / 2}$-cutoff function. Then everything is in the same spaces as before, and continues to be controlled by $c \theta$. The effect on $P$ and $\Phi$ is $P \equiv I$ and $\Phi \equiv 0$ on some neighborhood of $\partial B_{1 / 2}$. This implies $K_{j} \equiv 0$ there, too, hence we continue to have an equation of the form (11), but with nice boundary behaviour of $P$ and the $K_{j}$. Most importantly, $\psi \equiv 1$ on $B_{1 / 4}$ implies that on $B_{1 / 4}$ both equations coincide. 
By Lemma 4.2 below, the modified system has a solution on $B_{1 / 2}$ that is controlled as needed in the theorem. Hence we have found a solution $(\widetilde{A}, B)$ to the unmodified equation (11) on $B_{1 / 4}$. Transforming back, we find $(A, B)$ as asserted in part (i) of the theorem, with $A$ taking values in $G L(n)$ because of $A=(I+\widetilde{A}) P^{-1}$ and $\|\widetilde{A}\|_{L^{\infty}}<c \theta^{1 / 2}$. Hence the theorem is now proven up to Lemma 4.2 that follows.

Lemma 4.2 Assume we are given functions

$$
\begin{aligned}
P & \in W^{m, 2}\left(B^{2 m}, S O(n)\right), \\
K_{0} & \in W^{2-m, \frac{2 m}{m+1}, 1}\left(B^{2 m}\right), \\
K_{j} & \in W^{j+1-m, 2}\left(B^{2 m}\right) \quad \text { for } j \in\{1, \ldots, 2 m-2\}
\end{aligned}
$$

which are small in their respective spaces,

$$
\|d P\|_{W^{m-1,2}\left(B^{2 m}\right)}+\left\|K_{0}\right\|_{W^{2-m, \frac{2 m}{m+1}, 1}\left(B^{2 m}\right)}+\sum_{j=1}^{2 m-2}\left\|K_{j}\right\|_{W^{j+1-m, 2}\left(B^{2 m}\right)} \leq \theta
$$

for some $\theta$ to be chosen small enough. Assume that $P \equiv I$ (identity matrix) and $K_{j} \equiv 0$ $(0 \leq j \leq 2 m-2)$ hold on a neighborhood of $\partial B^{2 m}$. Then there exist

$$
\begin{aligned}
& A \in W^{m, 2} \cap L^{\infty}\left(B^{2 m}, \mathbb{R}^{n \times n}\right), \\
& B \in W^{2-m, 2}\left(B^{2 m}, \mathbb{R}^{n \times n} \otimes \wedge^{2} \mathbb{R}^{2 m}\right)
\end{aligned}
$$

such that the equation

$$
d \Delta^{m-1} A+\sum_{j=0}^{2 m-2}\left\langle D^{j} A, K_{j}\right\rangle+K_{0}=\delta B P
$$

holds in the sense of distributions. Moreover,

$$
\|A\|_{W^{m, 2}\left(B^{2 m}\right)}+\|A\|_{L^{\infty}\left(B^{2 m}\right)}+\|B\|_{W^{2-m, 2}\left(B^{2 m}\right)} \leq c \theta^{1 / 2} .
$$

Proof We are looking for $A$ and $B$ in the form of sums of derivatives

$$
\begin{aligned}
A & =\sum_{|\alpha| \leq m-2} \partial^{\alpha} A_{\alpha}, \quad A_{\alpha} \in W^{2 m-1, \frac{2 m}{2 m-1-|\alpha|}, 1}, \\
B & =\sum_{|\alpha| \leq m-2} \partial^{\alpha} B_{\alpha}, \quad B_{\alpha} \in W^{1, \frac{2 m}{2 m-1-|\alpha|}, 1} .
\end{aligned}
$$

Note that this even gives $A \in W^{m+1, \frac{2 m}{m+1}, 1}$ and $B \in W^{3-m, \frac{2 m}{m+1}, 1}$, but these spaces embed into those stated in the lemma. By the usual representation of negative Sobolev(-Lorentz) spaces, we find decompositions of $K_{0}, K_{1}, \ldots, K_{m-2}$ of the form

$$
\begin{aligned}
& K_{0}=\sum_{|\alpha| \leq m-2} \partial^{\alpha} K_{0}^{\alpha}, \quad K_{0}^{\alpha} \in L^{\frac{2 m}{m+1}, 1}, \\
& K_{j}=\sum_{|\alpha| \leq m-1-j} \partial^{\alpha} K_{j}^{\alpha}, \quad K_{j}^{\alpha} \in L^{2},
\end{aligned}
$$


such that $\sum_{\alpha}\left\|K_{j}^{\alpha}\right\| \leq c\left\|K_{j}\right\|$ in their respective norms. For $1 \leq j \leq m-2$, we then have (with certain integers $c_{\beta \gamma}$ )

$$
\begin{aligned}
\left\langle D^{j} A, K_{j}\right\rangle & =\sum_{|\alpha| \leq m-2} \sum_{|\beta| \leq m-1-j}\left\langle\partial^{\alpha} D^{j} A_{\alpha}, \partial^{\beta} K_{j}^{\beta}\right\rangle \\
& =\sum_{\alpha, \beta} \sum_{\gamma \leq \beta} \partial^{\gamma}\left[c_{\beta \gamma}\left\langle\partial^{\beta-\gamma} \partial^{\alpha} D^{j} A_{\alpha}, K_{j}^{\beta}\right\rangle\right] \\
& =: \sum_{\alpha, \beta} \sum_{\gamma \leq \beta} \partial^{\gamma}\langle A, K\rangle_{j, \alpha, \beta, \gamma},
\end{aligned}
$$

where here $\langle A, K\rangle_{j, \alpha, \beta, \gamma} \in W^{2 m-1-j-|\alpha|-|\beta|+|\gamma|, \frac{2 m}{2 m-1-|\alpha|}, 1} \cdot L^{2} \hookrightarrow L^{\frac{2 m}{j+|\beta|-|\gamma|}, 1} \cdot L^{2} \hookrightarrow$ $L^{\frac{2 m}{m+1-|\gamma|}, 1} \cdot L^{2} \hookrightarrow L^{\frac{2 m}{2 m-1-|\gamma|}}, 1$ and hence

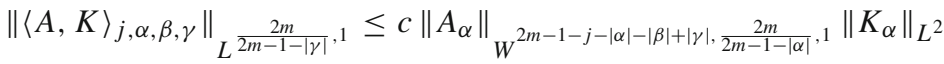

$$
\begin{aligned}
& \leq c \theta\|A\|_{W^{m+1, \frac{2 m}{m+1}, 1}}
\end{aligned}
$$

from the multiplication theorems.

Similarly, for $j=0$,

$$
\left\langle A, K_{0}\right\rangle=\sum_{|\alpha|,|\beta| \leq m-2} \sum_{\gamma \leq \beta} \partial^{\gamma}\langle A, K\rangle_{0, \alpha, \beta, \gamma}
$$

with $\langle A, K\rangle_{0, \alpha, \beta, \gamma} \in L^{\frac{2 m}{2 m-1-|\gamma|}, 1}$ (use $\partial^{\alpha} A_{\alpha} \in L^{\infty}$ ) and the estimate

$$
\left\|\langle A, K\rangle_{0, \alpha, \beta, \gamma}\right\|_{L^{\frac{2 m}{2 m-1-|\gamma|}, 1}} \leq c \theta\|A\|_{W^{m+1, \frac{2 m}{m+1}, 1}} .
$$

Now we turn our attention to $j \geq m-1$. This time, $K_{j}$ is a function, while $\partial^{\alpha} D^{j} A_{\alpha}$ may be only a distribution. Hence we have to shift derivatives in the opposite direction. Since $K_{j} \in W^{j+1-m, 2}$, we can shift at most $j+1-m$ derivatives; we will actually shift $\min \{|\alpha|, j+1-m\}$. We proceed as before. In the first case, $|\alpha| \leq j+1-m$, we have

$$
\left\langle\partial^{\alpha} D^{j} A_{\alpha}, K_{j}\right\rangle=\sum_{\gamma \leq \alpha} \partial^{\gamma} c_{\alpha \gamma}\left\langle D^{j} A_{\alpha}, \partial^{\alpha-\gamma} K_{j}\right\rangle .
$$

Since $D^{j} A_{\alpha} \in W^{2 m-1-j, \frac{2 m}{2 m-1-|\alpha|}, 1} \hookrightarrow L^{\frac{2 m}{j-|\alpha|}, 1}$ and $\partial^{\alpha-\gamma} K_{j} \in W^{j+1-m+|\gamma|-|\alpha|, 2}$ $\hookrightarrow L^{\frac{2 m}{2 m-j-1+|\alpha|-|\gamma|}}$, we have $\left\langle D^{j} A_{\alpha}, \partial^{\alpha-\gamma} K_{j}\right\rangle \in L^{\frac{2 m}{2 m-1-|\gamma|}, 1}$ and

$$
\left\|\left\langle D^{j} A_{\alpha}, \partial^{\alpha-\gamma} K_{j}\right\rangle\right\|_{L^{\frac{2 m}{2 m-1-|\gamma|}, 1}} \leq c \theta\|A\|_{W^{m+1, \frac{2 m}{m+1}, 1}} .
$$

In the second case, $j+1-m<|\alpha|$, we choose any $\beta \leq \alpha$ with $|\beta|=j+1-m$. Then

$$
\left\langle\partial^{\alpha} D^{j} A_{\alpha}, K_{j}\right\rangle=\sum_{\gamma \leq \beta} \partial^{\gamma}\left[c_{\beta \gamma}\left\langle\partial^{\alpha-\beta} D^{j} A_{\alpha}, \partial^{\beta-\gamma} K_{j}\right\rangle\right]
$$

with $\partial^{\alpha-\beta} D^{j} A_{\alpha} \in W^{m-|\alpha|, \frac{2 m}{2 m-1-|\alpha|}, 1} \hookrightarrow L^{\frac{2 m}{m-1}, 1}, \partial^{\beta-\gamma} K_{j} \in W^{|\gamma|, 2} \hookrightarrow L^{\frac{2 m}{m-|\gamma|}}$, and hence $\left\langle\partial^{\alpha-\beta} D^{j} A_{\alpha}, \partial^{\beta-\gamma} K_{j}\right\rangle \in L^{\frac{2 m}{2 m-1-|\gamma|}, 1}$, with the estimate (20) holding also in the second case. Combining (15), (17), (19), and (21), we find that

$$
\sum_{j=0}^{2 m-2}\left\langle D^{j} A, K_{j}\right\rangle=\sum_{|\gamma| \leq m-2} \partial^{\gamma}\langle A, K\rangle_{\gamma},
$$


where the terms on the right-hand side denoted symbolically by $\langle A, K\rangle_{\gamma}$ are estimated via (16), (18), and (20) by

$$
\left\|\langle A, K\rangle_{\gamma}\right\|_{L^{\frac{2 m}{2 m-1-|\gamma|}, 1}} \leq c \theta\|A\|_{W^{m+1, \frac{2 m}{m+1}, 1}} .
$$

Note that the single summand $K_{0}$ in (13) fits into the same scheme because of

$$
K_{0}=\sum_{|\gamma| \leq m-2} \partial^{\gamma} K_{0}^{\gamma} \quad \text { with } K_{0}^{\gamma} \in L^{\frac{2 m}{m+1}, 1} \hookrightarrow L^{\frac{2 m}{2 m-1-|\gamma|}, 1} .
$$

A similar treatment is necessary for the term $\delta B P$ in (13). We find

$$
\begin{aligned}
\delta B P & =\sum_{|\alpha| \leq m-2} \sum_{\gamma \leq \alpha} \partial^{\gamma}\left[\delta B_{\alpha} \partial^{\alpha-\gamma} P\right] \\
& =\sum_{|\gamma| \leq m-2} \partial^{\gamma}\left(\delta B_{\gamma} P+\sum_{\alpha>\gamma,|\alpha| \leq m-2} \delta B_{\alpha} \partial^{\alpha-\gamma} P\right) \\
& =: \sum_{|\gamma| \leq m-2} \partial^{\gamma}\left[\delta B_{\gamma} P+\langle\delta B, P\rangle_{\gamma}\right]
\end{aligned}
$$

with $\delta B_{\alpha} \partial^{\alpha-\gamma} P \in L^{\frac{2 m}{2 m-1-|\alpha|}, 1} \cdot W^{m-|\alpha|+|\gamma|, 2} \hookrightarrow L^{\frac{2 m}{2 m-1-|\alpha|}, 1} \cdot L^{\frac{2 m}{|\alpha|-|\gamma|}} \hookrightarrow L^{\frac{2 m}{2 m-1-|\gamma|}, 1}$, which also implies the estimate

$$
\begin{aligned}
\left\|\delta B_{\gamma} P+\langle\delta B, P\rangle_{\gamma}\right\|_{L^{\frac{2 m}{2 m-1-|\gamma|}, 1}} & \leq c\left(\sum_{|\alpha| \leq m-2}\left\|B_{\alpha}\right\|_{W^{1, \frac{2 m}{2 m-1-|\alpha|}, 1}}\right)\|d P\|_{W^{m-1,2}} \\
& \leq c \theta\|B\|_{W^{3-m, \frac{2 m}{m+1}, 1}} .
\end{aligned}
$$

Combining (22), (24), and (25), we rewrite (13) as

$$
\sum_{|\gamma| \leq m-2} \partial^{\gamma}[\underbrace{d \Delta^{m-1} A_{\gamma}+\langle A, K\rangle_{\gamma}+K_{0}^{\gamma}-\langle\delta B, P\rangle_{\gamma}-\delta B_{\gamma} P}_{=: G_{\gamma}}]=0 .
$$

Our approach to finding $A$ and $B$ is solving [...] $=0$ for every single $\gamma$, now that we have more unknown functions $A_{\gamma}, B_{\gamma}$ at our disposition. More precisely, we follow the idea from [8] to solving [...] $=0$ by splitting it in two equations $\delta[\ldots]=0$ and $d\left([\ldots] P^{-1}\right)=0$. This gives a system of equations

$$
\begin{aligned}
-\Delta^{m} A_{\gamma} & =\delta\left[\langle A, K\rangle_{\gamma}+K_{0}^{\gamma}-\langle\delta B, P\rangle_{\gamma}-\delta B_{\gamma} P\right], \\
d \delta B_{\gamma} & =d\left[\left\{d \Delta^{m-1} A_{\gamma}+\langle A, K\rangle_{\gamma}+K_{0}^{\gamma}-\langle\delta B, P\rangle_{\gamma}\right\} P^{-1}\right] .
\end{aligned}
$$

Note that this is a large system with two equations for every $\gamma$ with $|\gamma| \leq m-2$. We are going to choose suitable boundary conditions for each equation separately.

For each $\gamma$, we define the Banach space $E_{\gamma}$ to be $W^{2 m-1, \frac{2 m}{2 m-1-|\gamma|}, 1}$, but equipped with the norm

$$
\|f\|_{E_{\gamma}}:=\|f\|_{W^{2 m-1, \frac{2 m}{2 m-1-|\gamma|}, 1}}+\theta^{-1 / 2}\left\|d \Delta^{m-1} f\right\|_{L^{\frac{2 m}{2 m-1-|\gamma|}, 1}} .
$$

Then we let $\quad E:=\bigoplus_{|\gamma| \leq m-2} E_{\gamma}, \quad E^{\prime}:=\bigoplus_{|\gamma| \leq m-2} W^{2 m-1, \frac{2 m}{2 m-1-|\gamma|}, 1}, \quad L:=\bigoplus_{|\gamma| \leq m-1}$ $L^{\frac{2 m}{2 m-1-|\gamma|}}, 1, S:=\bigoplus_{|\gamma| \leq m-2} W^{1, \frac{2 m}{2 m-1-|\gamma|}}, 1$, and finally $R:=E \oplus S$. Writing $\mathbf{A}:=\left(A_{\gamma}\right)_{|\gamma| \leq m-2}$ and $\mathbf{B}:=\left(B_{\gamma}\right)_{|\gamma| \leq m-2}$, we are looking for $(\mathbf{A}, \mathbf{B}) \in R$ such that $(28)_{\gamma}$ and (29) $\gamma$ hold for all $\gamma$ with $|\gamma| \leq m-2$. 
We add boundary conditions and conditions on the $d B_{\gamma}$ in order to make the system uniquely solvable. For each $\gamma$, we want to solve the

Boundary value problem BVP Solve the system made of all (28) ${ }_{\gamma}$ and (29) $)_{\gamma}$ together with the Neumann boundary conditions

$$
\begin{aligned}
\frac{\partial \Delta^{j} A_{\gamma}}{\partial v} & =0 \quad \text { on } \partial B^{2 m}, \\
\int_{B^{2 m}} \Delta^{j} A_{\gamma} & =0
\end{aligned}
$$

for all $\gamma$ and all $j \in\{0, \ldots, m-1\}$, the additional equations

$$
d B_{\gamma}=0
$$

for all $\gamma$, as well as the boundary conditions

$$
\left(B_{\gamma}\right)_{N}=0 \quad \text { on } \partial B^{2 m}
$$

for all $\gamma$.

We will solve BVP by an iteration procedure. For any $(\mathbf{a}, \mathbf{b}) \in R$, let $T(\mathbf{a}, \mathbf{b})=:\left(\mathbf{a}^{\prime}, \mathbf{b}^{\prime}\right)$ be the solution of the system

$$
\begin{aligned}
-\Delta^{m} a_{\gamma}^{\prime} & =\delta\left[\langle a, K\rangle_{\gamma}+K_{0}^{\gamma}-\langle\delta b, P\rangle_{\gamma}-\delta b_{\gamma} P\right], \\
d \delta b_{\gamma}^{\prime} & =d\left[\left\{d \Delta^{m-1} a_{\gamma}+\langle a, K\rangle_{\gamma}+K_{0}^{\gamma}-\langle\delta b, P\rangle_{\gamma}\right\} P^{-1}\right], \\
\frac{\partial \Delta^{j} a_{\gamma}^{\prime}}{\partial v} & =0 \quad \text { on } \partial B^{2 m} \text { for } j \in\{0, \ldots, m-1\}, \\
\int_{B^{2 m}} \Delta^{j} a_{\gamma}^{\prime} & =0 \quad \text { for } j \in\{0, \ldots, m-1\}, \\
d b_{\gamma}^{\prime} & =0, \\
\left(b_{\gamma}^{\prime}\right)_{N} & =0 \quad \text { on } \partial B^{2 m},
\end{aligned}
$$

which must hold for all $\gamma$ with $|\gamma| \leq m-2$. We further split that problem and let $\left(\mathbf{a}^{1}, \mathbf{b}^{1}\right)$ be the solution of

$$
\begin{aligned}
-\Delta^{m} a_{\gamma}^{1} & =\delta K_{0}^{\gamma}, \\
d \delta b_{\gamma}^{1} & =d\left[K_{0}^{\gamma} P^{-1}\right],
\end{aligned}
$$

while $\left(\mathbf{a}^{2}, \mathbf{b}^{2}\right)$ is assumed to solve

$$
\begin{aligned}
-\Delta^{m} a_{\gamma}^{2} & =\delta\left[\langle a, K\rangle_{\gamma}-\langle\delta b, P\rangle_{\gamma}-\delta b_{\gamma} P\right], \\
d \delta b_{\gamma}^{2} & =d\left[\left\{d \Delta^{m-1} a_{\gamma}+\langle a, K\rangle_{\gamma}-\langle\delta b, P\rangle_{\gamma}\right\} P^{-1}\right],
\end{aligned}
$$

both with the last four conditions similar to the system for $\left(\mathbf{a}^{\prime}, \mathbf{b}^{\prime}\right)$.

By standard estimates for boundary value problems for differential forms (see [8] for a closely related system), the systems for $\left(\mathbf{a}^{1}, \mathbf{b}^{1}\right)$ and $\left(\mathbf{a}^{2}, \mathbf{b}^{2}\right)$ are uniquely solvable with 
estimates

$$
\begin{aligned}
& \left\|a_{\gamma}^{1}\right\|_{W^{2 m-1, \frac{2 m}{2 m-1-|\gamma|}, 1}} \leq c\left\|K_{0}^{\gamma}\right\|_{L^{2 m-1-|\gamma|}, 1}, \\
& \left\|b_{\gamma}^{1}\right\|_{W}^{1, \frac{2 m}{2 m-1-|\gamma|}, 1} \leq c\left\|K_{0}^{\gamma} P^{-1}\right\|_{L^{\frac{2 m}{2 m-1-|\gamma|}, 1}} \\
& \leq c\left\|K_{0}^{\gamma}\right\|_{L^{\frac{2 m}{2 m-1-|\gamma|}, 1}}, \\
& \left\|a_{\gamma}^{2}\right\|_{W^{2 m-1, \frac{2 m}{2 m-1-|\gamma|}, 1}} \leq c\left\|\langle a, K\rangle_{\gamma}-\langle\delta b, P\rangle_{\gamma}-d b_{\gamma} P\right\|_{L^{\frac{2 m}{2 m-1-|\gamma|}}, 1} \\
& \leq c \theta\left(\|\mathbf{a}\|_{E^{\prime}}+\|\mathbf{b}\|_{S}\right), \\
& \left\|b_{\gamma}^{2}\right\|_{W}^{1, \frac{2 m}{2 m-1-|\gamma|}, 1} \leq c\left\|d \Delta^{m-1} a_{\gamma}+\langle a, K\rangle_{\gamma}-\langle\delta b, P\rangle_{\gamma}\right\|_{L^{\frac{2 m}{2 m-1-|\gamma|}}, 1} \\
& \leq c\left\|d \Delta^{m-1} \mathbf{a}\right\|_{L}+c \theta\left(\|\mathbf{a}\|_{E^{\prime}}+\|\mathbf{b}\|_{S}\right),
\end{aligned}
$$

the latter two coming from (23) and (27). The estimates show that $T$ maps $R$ to itself.

Now we show that $T$ is a contraction on $R$. Let $(\mathbf{a}, \mathbf{b}),(\tilde{\mathbf{a}}, \tilde{\mathbf{b}}) \in R$. Since $\left(\mathbf{a}^{1}, \mathbf{b}^{1}\right)=$ $\left(\tilde{\mathbf{a}}^{1}, \tilde{\mathbf{b}}^{1}\right)$, we have estimates similar to (32) and (33),

$$
\begin{aligned}
\left\|\mathbf{a}^{\prime}-\tilde{\mathbf{a}}^{\prime}\right\|_{E^{\prime}} & =\left\|\mathbf{a}^{2}-\tilde{\mathbf{a}}^{2}\right\|_{E^{\prime}} \\
& \leq c \theta\left(\|\mathbf{a}-\tilde{\mathbf{a}}\|_{E^{\prime}}+\|\mathbf{b}-\tilde{\mathbf{b}}\|_{S}\right), \\
\left\|\mathbf{b}^{\prime}-\tilde{\mathbf{b}}^{\prime}\right\|_{S} & =\left\|\mathbf{b}^{2}-\tilde{\mathbf{b}}^{2}\right\|_{S} \\
& \leq c\left\|d \Delta^{m-1}(\mathbf{a}-\tilde{\mathbf{a}})\right\|_{L}+c \theta\left(\|\mathbf{a}-\tilde{\mathbf{a}}\|_{E^{\prime}}+\|\mathbf{b}-\tilde{\mathbf{b}}\|_{S}\right) \\
& \leq c \theta^{1 / 2}\|\mathbf{a}-\tilde{\mathbf{a}}\|_{E}+c \theta\left(\|\mathbf{a}-\tilde{\mathbf{a}}\|_{E^{\prime}}+\|\mathbf{b}-\tilde{\mathbf{b}}\|_{S}\right),
\end{aligned}
$$

from which we infer

$$
\left\|\left(\mathbf{a}^{\prime}, \mathbf{b}^{\prime}\right)-\left(\tilde{\mathbf{a}}^{\prime}, \tilde{\mathbf{b}}^{\prime}\right)\right\|_{R} \leq c \theta^{1 / 2}\|(\mathbf{a}, \mathbf{b})-(\tilde{\mathbf{a}}, \tilde{\mathbf{b}})\|_{R} .
$$

Hence $T$ is a contraction provided that $\theta$ has been chosen small enough. It therefore has a fixed point $(\mathbf{A}, \mathbf{B}) \in R$. The mapping $T$ has been set up in such a way that this fixed point solves BVP.

By estimates corresponding to (30)-(33) and $\mathbf{A}^{\prime}=\mathbf{A}, \mathbf{B}^{\prime}=\mathbf{B}$, we have

$$
\begin{aligned}
\|(\mathbf{A}, \mathbf{B})\|_{R} & =\left\|\mathbf{A}^{1}\right\|_{E}+\left\|\mathbf{B}^{1}\right\|_{S}+\left\|\mathbf{A}^{2}\right\|_{E}+\left\|\mathbf{B}^{2}\right\|_{S} \\
& \leq c \theta^{-1 / 2}\left\|K_{0}\right\|_{L}+c\left\|K_{0}\right\|_{L}+c \theta^{1 / 2}\left(\|\mathbf{A}\|_{E}+\|\mathbf{B}\|_{S}\right) \\
& \leq c \theta^{1 / 2}\left(1+\|(\mathbf{A}, \mathbf{B})\|_{R}\right) .
\end{aligned}
$$

Again if $\theta>0$ has been chosen small enough, then this implies $\|(\mathbf{A}, \mathbf{B})\|_{R} \leq c \theta^{1 / 2}$. Letting $A:=\sum_{|\alpha| \leq m-2} \partial^{\alpha} A_{\alpha}$ and $B:=\sum_{|\alpha| \leq m-2} \partial^{\alpha} B_{\alpha}$, we find that the norms in (14) are easily seen to be controlled by $\|(\mathbf{A}, \mathbf{B})\|_{R}$, thus (14) is proven.

Now that we have solved BVP, we need to show that (13) is solved by our $A$ and $B$, and we have already seen that this holds once we have proven that (28) and (29) (which are $\delta G_{\gamma}=0$ and $d\left(G_{\gamma} P^{-1}\right)=0$ ) together with the boundary data from BVP imply $G_{\gamma}=0$, with $G_{\gamma}$ defined in (27). Close to the boundary, the vanishing of $K_{j}$ and $d P$ simplifies the expression for $G_{\gamma}$, giving

$$
G_{\gamma}=d \Delta^{m-1} A_{\gamma}-\delta B_{\gamma}
$$

there. This means that the normal component of $G_{\gamma}$ vanishes, because we have Neumann boundary data for $\Delta^{m-1} A$, and $\left(B_{\gamma}\right)_{N}=0$ on $\partial B^{2 m}$ also implies $\left(\delta B_{\gamma}\right)_{N}=0$.

We have $G_{\gamma} \in L^{\frac{2 m}{2 m-1-|\gamma|}}, 1\left(\mathbb{R}^{n \times n} \otimes \wedge^{1} \mathbb{R}^{2 m}\right)$. A standard version of the Hodge decomposition (generalized to Lorentz spaces) says that $\delta G_{\gamma}=0$ on $B^{2 m}$ and $\left(G_{\gamma}\right)_{N}=0$ on $\partial B^{2 m}$ 
imply $G_{\gamma}=\delta C_{\gamma}$ for some $C_{\gamma} \in W^{1, \frac{2 m}{2 m-1-|\gamma|}, 1}\left(\mathbb{R}^{n \times n} \otimes \wedge^{2} \mathbb{R}^{2 m}\right)$ with $d C_{\gamma}=0$ on $B^{2 m}$ and $\left(C_{\gamma}\right)_{N}=0$ on $\partial B^{2 m}$. We combine the latter two equations with $d\left(G_{\gamma} P^{-1}\right)=0$ to the system

$$
d\left(\left(\delta C_{\gamma}\right) P^{-1}\right)=0, \quad d C_{\gamma}=0, \quad\left(\left(C_{\gamma}\right)_{N}\right)_{\mid \partial B^{2 m}}=0 .
$$

By Lemma 2.2, this implies $C_{\gamma}=0$ once $\theta$ has been chosen small enough to make $\left\|d\left(P^{-1}\right)\right\|=\|d P\|$ sufficiently small. Then also $G_{\gamma}=0$, which implies (13) and proves the lemma.

\section{Regularity}

From Theorem 4.1 (iii), we now easily infer that weak solutions of our differential equation (8) are continuous.

Theorem 5.1 Assume $U$ is a bounded open set in $\mathbb{R}^{2 m}$ and $u \in W^{m, 2}\left(U, \mathbb{R}^{n}\right)$ is a weak solution of (8) on $U$ with coefficient functions in the same spaces as in Theorem 4.1 (but without smallness condition). Then $u$ is continuous on $U$.

Proof Fix $x_{0} \in U$. For any $r>0$ small enough that $B_{r}\left(x_{0}\right) \subseteq U$ define $u_{r}: B^{2 m} \rightarrow$ $\mathbb{R}^{n}$ by $u_{r}(x):=u\left(x_{0}+r x\right)$. Then $u_{r}$ solves an equation on $B^{2 m}$ of the form (8), but with $V_{k}, w_{k}$ replaced by $V_{k, r}(x):=r^{2 m-2 k-2} V_{k}\left(x_{0}+r x\right)$ and $w_{k, r}(x):=r^{2 m-2 k-2} w_{k}\left(x_{0}+\right.$ $r x)$. We have $\left\|V_{k, r}\right\|_{W^{2 k+1-m, 2}\left(B^{2 m}\right)} \leq\left\|V_{k}\right\|_{W^{2 k+1-m-2}\left(B_{r}\left(x_{0}\right)\right)}$ and $\left\|w_{k, r}\right\|_{W^{2 k+2-m, 2}\left(B^{2 m}\right)} \leq$ $\left\|w_{k}\right\|_{W^{2 k+2-m-2}\left(B_{r}\left(x_{0}\right)\right)}$, both of which can be made arbitrarily small if $r>0$ is chosen small. Similar estimates hold for $\eta$ and $F$, and hence the smallness condition $\theta<\theta_{0}$ from Theorem 4.1 is fulfilled for the differential equation for $u_{r}$ once $r$ has been chosen sufficiently small. But then $u_{r}$ is continuous on $B_{1 / 16}$, which means $u$ is continuous on $B_{r / 16}\left(x_{0}\right)$.

Of course, in many special cases, we can expect much more than just continuity of weak solutions. As was proven in [3, Section 7], extrinsically or intrinsically polyharmonic maps are even smooth in $2 m$ dimensions, and, for a large class of equations, Hölder continuity of weak solutions implies smoothness. But here, we consider a very general equation with rather irregular coefficients, so maybe we cannot expect much regularity in general. Guo and Xiang [5] did prove Hölder continuity instead of just continuity in the fourth order case, and we learned of a recent preprint [6] where they achieve the same for our higher order case.

Funding Open Access funding enabled and organized by Projekt DEAL.

Open Access This article is licensed under a Creative Commons Attribution 4.0 International License, which permits use, sharing, adaptation, distribution and reproduction in any medium or format, as long as you give appropriate credit to the original author(s) and the source, provide a link to the Creative Commons licence, and indicate if changes were made. The images or other third party material in this article are included in the article's Creative Commons licence, unless indicated otherwise in a credit line to the material. If material is not included in the article's Creative Commons licence and your intended use is not permitted by statutory regulation or exceeds the permitted use, you will need to obtain permission directly from the copyright holder. To view a copy of this licence, visit http://creativecommons.org/licenses/by/4.0/.

\section{References}

1. Coifman, R., Lions, P.-L., Meyer, Y., Semmes, S.: Compensated compactness in Hardy spaces. J. Math. Pures Appl. 72, 247-286 (1993) 
2. Gastel, A.: The extrinsic polyharmonic map heat flow in the critical dimension. Adv. Geom. 6, 501-521 (2006)

3. Gastel, A., Scheven, C.: Regularity of polyharmonic maps in the critical dimension. Comm. Anal. Geom. 17, 185-226 (2009)

4. Goldstein, P., Zatorska-Goldstein, A.: Uhlenbeck's decomposition in Sobolev and Morrey-Sobolev spaces. Results Math. 73, (Art. 71) (2018)

5. Guo, C.-Y., Xiang, C.-L.: Regularity of solutions for a fourth-order elliptic system via conservation law. J. London Math. Soc. 101(2), 907-922 (2020)

6. Guo, C.-Y., Xiang, C.-L.: Regularity of weak solutions to higher order elliptic systems in critical dimensions. arXiv:2010.09149 (preprint)

7. Hélein, F.: Harmonic Maps. Conservation Laws and Moving Frames. Cambridge University Press (2002)

8. Lamm, T., Rivière, T.: Conservation laws for fourth order systems in four dimensions. Comm. Partial Diff. Eq. 33, 245-262 (2008)

9. O’Neil, R.: Convolution operators and $L(p, q)$ spaces. Duke Math. J. 30, 129-142 (1963)

10. Rivière, T.: Conservation laws for conformally invariant variational problems. Invent. Math. 168, 1-22 (2007)

11. Tartar, L.: Imbedding theorems of Sobolev spaces into Lorentz spaces. Boll. Unione Mat. Ital. Sez. B Artic. Ric. Mat. 8, 479-500 (1998)

12. Uhlenbeck, K.: Connections with $L^{p}$ bounds on curvature. Comm. Math. Phys. 83, 31-42 (1982)

13. Ziemer, W.: Weakly Differentiable Functions. Sobolev Spaces and Functions of Bounded Variation. Graduate Texts in Mathematics, vol. 120. Springer, New York (1989)

Publisher's Note Springer Nature remains neutral with regard to jurisdictional claims in published maps and institutional affiliations. 